\title{
THE COMPUTATION OF SEXTIC FIELDS WITH A CUBIC SUBFIELD AND NO QUADRATIC SUBFIELD
}

\author{
M. OLIVIER
}

\begin{abstract}
We describe six tables of sixth-degree fields $K$ containing a cubic subfield $k$ and no quadratic subfield: one for totally real sextic fields, one for sextic fields with four real places, two for sextic fields with two real places, and two for totally imaginary sextic fields (depending on whether the cubic subfield is totally real or not). The tables provide for each possible discriminant $d_{K}$ of $K$ a quadratic polynomial which defines $K / k$, the discriminant of the cubic subfield and the Galois group of a Galois closure $N / \mathbb{Q}$ of $K / \mathbb{Q}$.
\end{abstract}

\section{INTRODUCTION}

In a previous paper [3] with A.-M. Bergé and J. Martinet, we described relative methods for finding sextic fields with a quadratic subfield up to a given bound on the discriminant. These methods were inspired by general considerations on a geometric approach to this subject explained by J. Martinet in [11] (see also [7]). They provide algorithmic tools for constructing extensive tables of number fields of given degree and signature in the relative case. Here we develop these tools to compute tables of sextic fields with a cubic subfield and no quadratic subfield (those fields with both a cubic and a quadratic subfield are presented in the tables mentioned above [3]).

We first require tables of totally real and complex cubic fields $k$, their integral bases, and the decomposition of the rational primes in $k$. In $\S 3$ we provide an outline of the computational methods that we employed.

The first step relies on geometric methods. Given a signature, a cubic field $k$, and a bound $M$, it consists of building a list of quadratic polynomials over $k$ such that any sextic field $K / \mathbb{Q}$ containing $k$ with absolute value of discriminant less than $M$ is defined by some of these polynomials. The bound $M$ depends on the signature: $7 \cdot 10^{7}$ for totally real sextic fields, $2 \cdot 10^{7}$ for sextic fields with four real places, $16 \cdot 10^{6}$ for sextic fields with two real places and a totally real cubic subfield, $3 \cdot 10^{6}$ for sextic fields with two real places and a complex cubic subfield, $5 \cdot 10^{7}$ for totally imaginary sextic fields with a totally real cubic subfield, and $3 \cdot 10^{6}$ for totally imaginary sextic fields with a complex cubic subfield. This is discussed in $\S 4$.

In the second step, we remove the reducible polynomials and those which define a sextic field containing a quadratic subfield (these fields are in the tables

Received July 11, 1990; revised February 25, 1991.

1991 Mathematics Subject Classification. Primary 11R21, 11Y40, 11 R32. 
described in [3]); using approximations of the roots, we then compute by localization the relative discriminant $\mathfrak{d}_{K / k}$ of $K / k$; a relative integral basis might not exist (§5).

The third step is devoted to testing isomorphisms between sextic fields having the same relative discriminant; in our case, this can be done by two differents methods which are described in $\S 6$. We choose from among the quadratic polynomials defining the same sextic field one which gives the smallest index.

In the fourth step $(\S 7)$ we determine the Galois group of a Galois closure $N$ of $K$ over $\mathbb{Q}$; the classification of such transitive groups of degree six has been done, e.g., by G. Butler and J. McKay ([4]; see also [12]).

Finally, in $\S 8$, we provide some comments on the tables. By class field theory we obtain results on imprimitive quartic fields which concern the parity of the class number; we also list the minimal discriminants with given infinite Frobenius for the five isomorphism classes of transitive groups of sixth degree that we consider in this paper.

In order to complete this work, we intend, in a forthcoming paper [13], to use the methods of Pohst [14], to compute extensive tables of primitive sextic fields for all signatures.

\section{Notation}

We denote by $K$ a quadratic extension of a cubic field $k ;\left(r_{1}, r_{2}\right)$ is the signature of $K ; \mathbb{Z}_{K}$ (resp. $\mathbb{Z}_{k}$ ) is the ring of integers of $K$ (resp. $k$ ); $N$ is a Galois closure of $K / \mathbb{Q} ; \mathfrak{d}_{K / k}$ is the ideal discriminant of $K / k ; d_{K}$ is the absolute discriminant of $K / \mathbb{Q}$; and $d_{P}$ is the discriminant of the polynomial $P$.

$v_{\mathfrak{p}}$ is the valuation associated with the prime ideal $\mathfrak{p}$ of $k$ (same notation with capital letters $v_{\mathfrak{P}}$, for $\mathfrak{P}$ in $K$ ); $\operatorname{Tr}_{k / \mathbb{Q}}\left(\right.$ resp. $\operatorname{Tr}_{K / k}$ ) and $N_{k / \mathbb{Q}}$ (resp. $N_{K / k}$ ) are respectively the trace and the norm of $k / \mathbb{Q}$ (resp. $\left.K / k\right)$.

The cubic field $k / \mathbb{Q}$ of discriminant $d_{k}$ is defined by a primitive element $\alpha ;\{1, \alpha, \beta\}$ is an integral basis of $\mathbb{Z}_{k}, Q$ (resp. $R$ ) the minimal polynomial of $\alpha$ (resp. $\beta$ ) (in most of the cases, we have $\left.\beta=\alpha^{2}\right) ;\left(\alpha, \alpha^{\prime}, \alpha^{\prime \prime}\right)$ (resp. $\left.\left(\beta, \beta^{\prime}, \beta^{\prime \prime}\right)\right)$ are the conjugates of $\alpha$ (resp. $\beta$ ) under the conjugacy of $k / \mathbb{Q}$.

The sextic field $K$ is defined by a primitive element $\theta$ over $k$; its minimal polynomial is $P(x)=x^{2}-a x+b \in \mathbb{Z}_{k}[x] ; \theta_{1}$ and $\theta_{2}$ are the roots of $P$; we let $P^{\prime}$ (resp. $P^{\prime \prime}$ ) be the polynomial with coefficients $a^{\prime}$ and $b^{\prime}$ (resp. $a^{\prime \prime}$ and $b^{\prime \prime}$ ), with roots $\theta_{1}^{\prime}$ and $\theta_{2}^{\prime}$ (resp. $\theta_{1}^{\prime \prime}$ and $\theta_{2}^{\prime \prime}$ ) in an algebraic closure of $\mathbb{Q}$.

\section{Computation IN CUBIC FIELDS}

If $K$ is a sextic field containing a cubic subfield $k$, we have

$$
\left|d_{K}\right|=d_{k}^{2} N_{k / \mathbb{Q}}\left(\mathfrak{d}_{K / k}\right) ;
$$

thus, to construct tables of sextic fields having a cubic subfield, and with discriminant up to a given bound $M$, we need tables of cubic fields with discriminant up to $\sqrt{M}$.

Such tables of cubic fields exist (see, for instance, $[2,5,8,10]$ for totally real cubic fields, and $[1,15]$ for complex cubic fields).

For our purpose, we use the following method: first search for polynomials $Q(x)=x^{3}-c_{1} x^{2}+c_{2} x-c_{3}$ in $\mathbb{Z}[x]$ defining $k / \mathbb{Q}$, using the inequality of 
Corollary 2.9 in [11]. Given such a polynomial, using Theorem 2 in [9], we compute the discriminant $d_{k}$. Then we test isomorphisms between cubic fields having the same discriminant. Finally, the results in Chapter 3 of [16] allow us to find an integral basis of $\mathbb{Z}_{k}$ of the form $(1, \alpha, \beta)$, with $Q(\alpha)=0$.

In the paper [9] mentioned above, Theorem 1 describes the decomposition of the primes in $\mathbb{Z}_{k}$. In fact, in $\S 5$, we make the effective computations required to choose an explicit representation of the prime ideals $\mathfrak{p}$ in $\mathbb{Z}_{k}$ above a prime rational $p$ so as to be able, given an integer $x \in \mathbb{Z}_{k}$ and a prime ideal $\mathfrak{p}$, to compute $v_{\mathfrak{p}}(x)$.

The following lemmas can be used to carry out these tasks.

Lemma 1. For every prime ideal $\mathfrak{p}$ above $p$ in $\mathbb{Z}_{k}$, there exists a $\gamma$ in $\mathbb{Z}_{k}$ such that $\mathfrak{p}=p \mathbb{Z}_{k}+\gamma \mathbb{Z}_{k}$ with the following properties:

(1) $v_{\mathfrak{p}}(\gamma)=1$;

(2) if $\mathfrak{p}^{\prime}$ is another prime ideal above $p, v_{\mathfrak{p}^{\prime}}(\gamma)=0$.

(When $p$ is inert, we choose $\gamma=p$.)

Proof. If $p$ does not divide the index $\left[\mathbb{Z}_{k}: \mathbb{Z}[\alpha]\right]$, by a classical result, the prime decomposition $\bmod p$ of the minimal polynomial $Q$ of $\alpha$ gives the decomposition of the prime number $p$ in $\mathbb{Z}_{k}$; if $\bar{Q}(x)=\prod_{1 \leq i \leq g} \bar{\varphi}(x)^{e_{i}} \bmod p$, we have $p \mathbb{Z}_{k}=\prod_{1 \leq i \leq g} \mathfrak{p}_{i}^{e_{i}}$, with $\mathfrak{p}_{i}=p \mathbb{Z}_{k}+\gamma \mathbb{Z}_{k}$, the degree of $\bar{\varphi}$ is the degree of $\mathfrak{p}_{i}$, and if $e_{i}>1$, then $\gamma=\varphi(\alpha)$, otherwise, $\gamma=\varphi(\alpha)$ or $\varphi(\alpha)+p$ according to the $p$-valuation of $\varphi(\alpha)$.

If $p$ divides the index, it is easy to prove that we can choose $\varepsilon_{1}$ and $\varepsilon_{2}$ in $\{0,1\}$ and $u$ in $\mathbb{Z}$ such that $\gamma=\varepsilon_{1} \alpha+\varepsilon_{2} \beta+u$, depending on the factorization $\bmod p$ of $Q$ and $R$.

More precisely, the results are (note that in this situation, $p$ divides $d_{Q}$ and $d_{R}$, and the minimal polynomials of $\alpha$ and $\beta$ have a multiple root $\bmod p$ ):

If $p \mathbb{Z}_{k}=\mathfrak{p}^{3}$, then $Q($ resp. $R)$ has a triple root $t\left(\right.$ resp. $\left.t^{\prime}\right) \bmod p$; thus, we can take $\gamma=\alpha-t$ if $v_{\mathfrak{p}}(\alpha-t)=1$, and $\gamma=\beta-t^{\prime}$ otherwise.

If $p \mathbb{Z}_{k}=\mathfrak{p}_{1}^{2} \mathfrak{p}_{2}$, then if $Q$ has a simple root $t_{1}$ and a double root $t_{2} \bmod p$, we have $\mathfrak{p}_{2}=\left(p, \alpha-t_{1}\right)$ or $\left(p, \alpha-t_{1}+p\right) ; \mathfrak{p}_{1}=\left(p, \alpha-t_{2}\right)$ if $v_{\mathfrak{p}_{1}}\left(\alpha-t_{2}\right)=1$; if $v_{\mathfrak{p}_{1}}\left(\alpha-t_{2}\right)>1$, then $\mathfrak{p}_{1}=\left(p, \beta-t^{\prime}\right)$ if $t^{\prime}$ is a double root of $R \bmod p$, and $\mathfrak{p}_{1}=\left(p, \alpha+\beta-t_{2}-t^{\prime}\right)$ if $t^{\prime}$ is of order 3 . In the case when $Q$ has a triple root, then $R$ has a single and a double root, so we exchange $Q$ and $R$ in the above discussion.

If $p \mathbb{Z}_{k}=\mathfrak{p}_{1} \mathfrak{p}_{2}$ with $\mathfrak{p}_{2}$ of degree 2 , then if $Q$ has a simple root $t_{1}$ and a double root $t_{2} \bmod p$, then $\mathfrak{p}_{1}=\left(p, \alpha-t_{1}\right)$ or $\left(p, \alpha-t_{1}+p\right)$; if $v_{\mathfrak{p}_{2}}\left(\alpha-t_{2}\right)=1$, then $\mathfrak{p}_{2}=\left(p, \alpha-t_{2}\right)$; if not, $\mathfrak{p}_{2}=\left(p, \beta-t^{\prime}\right)$ if $t^{\prime}$ is a root of order 2 of $R$, and $\mathfrak{p}_{2}=\left(p, \alpha+\beta-t_{2}-t^{\prime}\right)$ if $t^{\prime}$ is of order 3. If $Q$ has a triple root, exchange $Q$ and $R$ as above.

Finally, if $p \mathbb{Z}_{k}=\mathfrak{p}_{1} \mathfrak{p}_{2} \mathfrak{p}_{3}$, the only case is: $Q$ has a simple root $t_{1}$ and a double root $t_{2} \bmod p$ (idem for $R$ with $t_{1}^{\prime}$ and $\left.t_{2}^{\prime}\right)$; let $\mathfrak{p}_{1}=\left(p, \alpha-t_{1}\right)$ or $\left(p, \alpha-t_{1}+p\right)$ and choose $\mathfrak{p}_{2}=\left(p, \beta-t_{1}^{\prime}\right)$ or $\left(p, \beta-t_{1}^{\prime}+p\right)$ and $\mathfrak{p}_{3}=$ $\left(p, \alpha+\beta-t_{2}-t_{2}^{\prime}\right)$ or $\left(p, \alpha+\beta-t_{2}-t_{2}^{\prime}+p\right)$.

Lemma 2. Let $\mathfrak{p}=(p, \gamma)$ be a prime ideal of $\mathbb{Z}_{k}$ of degree $1, \gamma$ as in Lemma 1 ; let $x=x_{1}+x_{2} \alpha+x_{3} \beta \quad\left(x_{i}\right.$ in $\left.\mathbb{Z}\right)$ be an integer of $\mathbb{Z}_{k}$, and $a$ (resp. $\left.b\right)$ in $\mathbb{Z}$ such that $\alpha \equiv a \bmod \mathfrak{p}($ resp. $\beta \equiv b)$; finally, let $y=x N_{k / \mathbb{Q}}(\gamma) / p \gamma$. Then, if $v_{p}\left(x_{1}+x_{2} a+x_{3} b\right)$ is $\geq 1, y$ belongs to $\mathfrak{p}$ and $v_{\mathfrak{p}}(x)=1+v_{\mathfrak{p}}(y)$. 
Proof. Clearly, $v_{p}\left(x_{1}+x_{2} a+x_{3} b\right) \geq 1$ is equivalent to $v_{\mathfrak{p}}(x) \geq 1$; moreover, $(x / \gamma)=\left(p y / N_{k / \mathbb{Q}}(\gamma)\right)$ and the hypothesis on $\gamma$ in Lemma 1 imply the result.

Now we have an algorithm to compute $v_{\mathfrak{p}}(x)$ for $x \in \mathbb{Z}_{k}$ and $\mathfrak{p}$ a prime ideal of degree $f$ and ramification index $e$ :

(1) write $x=n x^{\prime}$ with $n \in \mathbb{Z}$ and $x^{\prime}=x_{1}^{\prime}+x_{2}^{\prime} \alpha+x_{3}^{\prime} \beta$ such that $\operatorname{gcd}\left(x_{1}^{\prime}, x_{2}^{\prime}, x_{3}^{\prime}\right)=1$

(2) compute $v_{\mathfrak{p}}(n)=e v_{p}(n)$;

(3) if $f=3$, then $v_{\mathrm{p}}\left(x^{\prime}\right)=0$;

(4) else if $f=2$, then $p \mathbb{Z}_{k}=\mathfrak{p p}^{\prime}$ and $v_{\mathfrak{p}}\left(x^{\prime}\right)=\frac{1}{2}\left(v_{p}\left(N_{k / \mathbb{Q}}\left(x^{\prime}\right)\right)-v_{\mathfrak{p}^{\prime}}\left(x^{\prime}\right)\right)$;

(5) else if $f=1$ and $e=3$, then $v_{\mathfrak{p}}\left(x^{\prime}\right)=v_{p}\left(N_{k / \mathbb{Q}}\left(x^{\prime}\right)\right)$;

(6) else compute $a$ and $b$ such that $\alpha \equiv a$ and $\beta \equiv b \operatorname{modp}$;

we have $v_{\mathfrak{p}}\left(x^{\prime}\right) \geq 1$ if and only if $v_{p}\left(x_{1}^{\prime}+x_{2}^{\prime} a+x_{3}^{\prime} b\right) \geq 1$; in the case of $v_{\mathfrak{p}}\left(x^{\prime}\right) \geq 1$, Lemma 2 gives a recursion formula, $v_{\mathfrak{p}}\left(x^{\prime}\right)=1+v_{\mathfrak{p}}\left(x^{\prime \prime}\right)$ with $x^{\prime \prime}=\left(x^{\prime} / p\right)\left(N_{k / \mathbb{Q}}(\gamma) / \gamma\right)$.

\section{COMPUTATIONAL METHODS FOR SEARCHING FOR POLYNOMIALS}

General method. ${ }^{1}$ We give here general relative methods for constructing tables of irreducible polynomials $P(x)=x^{m}-a_{1} x^{m-1}+\cdots+(-1)^{m} a_{m} \in \mathbb{Z}_{k}[x]$ which define a relative extension $K / k$ of degree $m$, where $k$ is a number field of degree $n^{\prime} \quad\left(n=m n^{\prime}\right)$. The notation is the following: $\theta$ is a primitive element $(K=k(\theta)),\left(\theta_{1}, \ldots, \theta_{m}\right)$ are the roots of $P$ in $\mathbb{C}$; if $c$ is in $k$, $\left(c=c^{(1)}, \ldots, c^{\left(n^{\prime}\right)}\right)$ are the conjugates of $c$ under the conjugacy of $k / \mathbb{Q}$. By extension, $\left(P=P^{(1)}, \ldots, P^{\left(n^{\prime}\right)}\right)$ denote the polynomials whose coefficients are the $\left(a_{i}^{(h)}\right)$ 's, and $\left(\theta_{j}^{(h)}\right)$ are their roots in $\mathbb{C} .\left(1=\alpha_{1}, \ldots, \alpha_{n^{\prime}}\right)$ is an integral basis of $\mathbb{Z}_{k}$. Finally, by analogy with the formulation of M. Pohst in [14], we define

$$
\begin{aligned}
S_{l}^{(h)}(\theta) & =\sum_{i=1}^{m}\left(\theta_{i}^{(h)}\right)^{l}, & S_{l}(\theta) & =\sum_{h=1}^{n^{\prime}} S_{l}^{(h)}(\theta), \\
T_{l}^{(h)}(\theta) & =\sum_{i=1}^{m}\left|\theta_{i}^{(h)}\right|^{l}, & T_{l}(\theta) & =\sum_{h=1}^{n^{\prime}} T_{l}^{(h)}(\theta),
\end{aligned}
$$

for $1 \leq l \leq m$. The basic tool of the method is Theorem 2.8 in [11]:

Theorem. There exists an element $\theta \in \mathbb{Z}_{K}$ such that $K=k(\theta)$ and

$$
T_{2}(\theta) \leq \frac{1}{m} \sum_{h=1}^{n^{\prime}}\left|a_{1}^{(h)}\right|^{2}+\gamma_{n-n^{\prime}}\left(\frac{\left|d_{K}\right|}{m^{n^{\prime}}\left|d_{k}\right|}\right)^{1 /\left(n-n^{\prime}\right)},
$$

where $\gamma_{q}$ is the Hermite constant in dimension $q$, and $d_{k}\left(\right.$ resp. $\left.d_{K}\right)$ is the absolute discriminant of $k$ (resp. $K$ ).

Moreover, $\theta$ is arbitrary modulo $\mathbb{Z}_{k}$.

In the following, we write $c_{1}=\frac{1}{m} \sum_{h=1}^{n^{\prime}}\left|a_{1}^{(h)}\right|^{2}$ and

$$
c(M)=\gamma_{n-n^{\prime}}\left(\frac{M}{m^{n^{\prime}}\left|d_{k}\right|}\right)^{1 /\left(n-n^{\prime}\right)},
$$

${ }^{1}$ I am grateful to the referee for a number of suggestions which led to improvements in the first draft of this section. 
where $M$ is the bound for the absolute discriminant $\left|d_{K}\right|$ of the fields $K$ we are looking for, and $c_{2}(M)=c_{1}+c(M)$; so, the above inequality becomes $T_{2}(\theta) \leq c_{2}(M)$.

We now give inequalities to bound the $a_{i}$ 's.

Changing $\theta$ in $\theta+\lambda$, with $\lambda \in \mathbb{Z}_{k}$, and using the fact that $a_{1}=\sum_{1 \leq j \leq n^{\prime}} a_{1, j} \alpha_{j}$ $=\operatorname{Tr}_{K / k}(\theta)\left(a_{1, j} \in \mathbb{Z}\right)$, we see that we can choose the $a_{1, j}$ 's modulo $m$.

Now, we fix $a_{1}$ (hence $c_{1}$ ) among the $m^{n^{\prime}}$ possible values and search for the last coefficient $a_{m}=\sum_{1 \leq j \leq n^{\prime}} a_{m, j} \alpha_{j}$.

The inequality between arithmetic and geometric means yields the following result:

$$
\left|a_{m}^{(h)}\right|^{2}=\prod_{i=1}^{m}\left|\theta_{i}^{(h)}\right|^{2} \leq\left(\frac{1}{m} \sum_{i=1}^{m}\left|\theta_{i}^{(h)}\right|^{2}\right)^{m}=\frac{1}{m^{m}}\left(T_{2}^{(h)}(\theta)\right)^{m},
$$

and therefore the inequality

$$
\sum_{h=1}^{n^{\prime}}\left|a_{m}^{(h)}\right|^{2} \leq \frac{1}{m^{m}} \sum_{h=1}^{n^{\prime}}\left(T_{2}^{(h)}(\theta)\right)^{m} \leq \frac{1}{m^{m}} T_{2}(\theta)^{m} .
$$

Finally, writing $c_{3}(M)=c_{2}(M)^{m} / m^{m}$, and applying the above theorem, we obtain

$$
\sum_{h=1}^{n^{\prime}}\left|a_{m}^{(h)}\right|^{2} \leq c_{3}(M)
$$

Let $b_{i, j}=\sum_{1 \leq h \leq n^{\prime}} \alpha_{i}^{(h)} \overline{\alpha_{j}^{(h)}}$; we have to compute all $\left(a_{m, j}\right)_{1 \leq j \leq n^{\prime}} \in \mathbb{Z}^{n^{\prime}}$ subject to

$$
\sum_{1 \leq i, j \leq n^{\prime}} b_{i, j} a_{m, i} a_{m, j} \leq c_{3}(M)
$$

where $\sum_{1 \leq i, j \leq n^{\prime}} b_{i, j} x_{i} x_{j}$ is a positive definite quadratic form. This can be done using the Fincke-Pohst algorithm (see [6]). Note that this method will be applied for the other $a_{i}$ 's.

We search now for $a_{2}$.

For $1 \leq h \leq n^{\prime}$, we have, $a_{2}^{(h)}=\frac{1}{2}\left(\left(S_{1}^{(h)}(\theta)\right)^{2}-S_{2}^{(h)}(\theta)\right)$, and

$$
\sum_{1 \leq h \leq n^{\prime}}\left|S_{2}^{(h)}(\theta)\right|^{2} \leq T_{2}(\theta)^{2} \leq c_{2}(M)^{2}
$$

and applying the above algorithm, we calculate the integer points into this ellipsoid.

To finish, we need to find the other $a_{i}$ 's for $3 \leq i<m$.

We make use of a method due to $M$. Pohst which allows us to compute a bound for the $T_{i}(\theta)$, knowing $a_{1}, a_{2}$, and $a_{m}$ (cf. Theorem 4 in [14]). Given these bounds, we proceed by recursion on $i$ as follows:

We suppose that we know $a_{3}, \ldots, a_{i-1}$; then Newton's formula gives

$$
a_{i}^{(h)}=\frac{1}{i}\left(\sum_{j=1}^{i-1}(-1)^{j-1} a_{i-j}^{(h)} S_{j}^{(h)}(\theta)+(-1)^{i-1} S_{i}^{(h)}(\theta)\right),
$$

and we have

$$
\sum_{h=1}^{n^{\prime}}\left|S_{i}^{(h)}(\theta)\right|^{2} \leq T_{i}(\theta)^{2}
$$

so, we are able to finish as for the first $a_{i}$ 's. 
Application to sextic fields. Let us apply the previous method to the totally real sextic fields containing the cubic subfield $k=\mathbb{Q}(\alpha)$, with discriminant 49 , the minimal polynomial of $\alpha$ being $x^{3}+x^{2}-2 x-1$, whose roots are $\alpha_{2}^{(1)}=$ $1.246 \ldots, \alpha_{2}^{(2)}=-0.445 \ldots, \alpha_{2}^{(3)}=-1.801 \ldots$. A $\mathbb{Z}$-basis of $\mathbb{Z}_{k}$ is $\left(1, \alpha_{2}, \alpha_{3}\right)$, with $\alpha_{3}=\alpha_{2}^{2}$, and we have $\alpha_{3}^{(1)}=1.554 \ldots, \alpha_{3}^{(2)}=0.198 \ldots, \alpha_{3}^{(3)}=3.246 \ldots$.

We are looking for polynomials $P(x)=x^{2}-a_{1} x+a_{2} \in \mathbb{Z}_{k}[x]$ such that $K=k(\theta)$ and $P$ is the minimal polynomial of $\theta$.

We take $M=7 \cdot 10^{7}$; first, we have $c(M)=70.949 \ldots$; we choose $a_{1}$ among the eight possible values: $0,1, \alpha_{2}, \alpha_{2}^{2}, 1+\alpha_{2}, 1+\alpha_{2}^{2}, \alpha_{2}+\alpha_{2}^{2}, 1+\alpha_{2}+\alpha_{2}^{2}$.

For $a_{1}=0, c_{3}(M)=1258.446 \ldots$, and we have to solve the inequality

$$
3\left(x_{1}-\frac{1}{3} x_{2}+\frac{5}{3} x_{3}\right)^{2}+\frac{14}{3}\left(x_{2}-\frac{1}{2} x_{3}\right)^{2}+\frac{7}{2} x_{3}^{2} \leq 1258.446 \ldots,
$$

with $\left(x_{1}, x_{2}, x_{3}\right) \in \mathbb{Z}^{3}$. We find 27294 vectors; among those vectors, only 1001 give totally real irreducible polynomials satisfying the inequality of the previous theorem.

Irreducibility of quadratic polynomials. Given a polynomial $P(x)=x^{2}-a x+b \in$ $\mathbb{Z}_{k}$, we need to test whether it is irreducible over $k[x]$. To do this, we compute approximations of the roots $\theta_{1}, \theta_{2}, \theta_{1}^{\prime}, \theta_{2}^{\prime}, \theta_{1}^{\prime \prime}, \theta_{2}^{\prime \prime}$; for all reasonable triples (depending on the signature) we test if $\theta_{i}+\theta_{j}^{\prime}+\theta_{k}^{\prime \prime}, \theta_{i} \theta_{j}^{\prime}+\theta_{i} \theta_{k}^{\prime \prime}+\theta_{j}^{\prime} \theta_{k}^{\prime \prime}$, and $\theta_{i} \theta_{j}^{\prime} \theta_{k}^{\prime \prime}$ are in $\mathbb{Z}$. If they are, we guess the possible root of $P$ in $\mathbb{Z}_{k}$ and verify whether or not it is in $\mathbb{Z}_{k}$.

\section{RELATIVE DISCRIMINANT}

Let $P$ be a polynomial in the preceding list with discriminant $d_{P}$; for every prime number $p$ dividing $N_{k / \mathbb{Q}}\left(d_{P}\right)$, and for each prime ideal $\mathfrak{p}$ of $k$ above $p$, we have to compute $v_{\mathfrak{p}}\left(\mathfrak{d}_{K / k}\right)$.

First of all, since $d_{P}=\mathfrak{f}^{2} \mathfrak{d}_{K / k}$, where $\mathfrak{f}$ is the index of $\mathbb{Z}_{k}[\theta]$ in $\mathbb{Z}_{K}, v_{\mathfrak{p}}\left(\mathfrak{d}_{K / k}\right)$ and $v_{\mathfrak{p}}\left(d_{P}\right)$ are simultaneously even or odd and $v_{\mathfrak{p}}\left(\mathfrak{d}_{K / k}\right)$ is zero as soon as $v_{\mathfrak{p}}\left(d_{P}\right)$ is. So, we have only to deal with those $\mathfrak{p}$ for which $v_{\mathfrak{p}}\left(d_{P}\right)$ is not zero.

We denote by $\pi$ a uniformizing parameter at $\mathfrak{p}$, and by $e_{0}$ the absolute ramification index of $(2)$ in $\mathbb{Z}_{k}$; then, we use local computations.

We write $d_{P}=\pi^{2 l} x$, with $x \in k$ and $v_{\mathfrak{p}}(x)=0$ or 1 . The effective calculation of $\mathfrak{d}_{K / k}$ is based on the following two results:

Proposition 1. If $\mathfrak{p}$ does not divide (2), then $v_{\mathfrak{p}}\left(\mathfrak{d}_{K / k}\right)=0$ or 1 according to whether $v_{\mathfrak{p}}(x)=0$ or 1 . If $\mathfrak{p}$ divides (2) and $v_{\mathfrak{p}}(x)=1$, then $v_{\mathfrak{p}}\left(\mathfrak{d}_{K / k}\right)=$ $1+2 e_{0}$.

The proof is obvious: if $v_{\mathfrak{p}}(x)=1$, the polynomial $X^{2}-x$ is an Eisenstein polynomial with discriminant $4 x$; if $v_{\mathfrak{p}}(x)=0$ and if $\mathfrak{p}$ does not divide (2), the discriminant of the polynomial $X^{2}-x$ has valuation zero in $\mathfrak{p}$, and therefore $v_{\mathfrak{p}}\left(\mathfrak{d}_{K / k}\right)=0$.

The next proposition deals with the remaining case when $\mathfrak{p}$ divides (2) and $v_{\mathfrak{p}}(x)=0$.

Proposition 2. Let $m=\max \left\{n \mid x=\square \bmod \mathfrak{p}^{n}\right\}$; if $m \geq 2 e_{0}$, we have $v_{\mathfrak{p}}\left(\mathfrak{d}_{K / k}\right)$ $=0$, and in the others cases, $m$ is odd and $v_{\mathfrak{p}}\left(\mathfrak{d}_{K / k}\right)=2 e_{0}-(m-1)$. 
The proof consists of an elementary study of the ramification groups together with some results "à la Hecke” concerning quadratic extensions.

It is easy to deduce from the above propositions an algorithm to compute $v_{\mathfrak{p}}\left(\mathfrak{d}_{K / k}\right)$ for all prime ideals $\mathfrak{p}$ :

Step 1. If $v_{\mathfrak{p}}\left(d_{P}\right)=0$, then $v_{\mathfrak{p}}\left(\mathfrak{d}_{K / k}\right)=0$.

Step 2. Else, if $v_{\mathfrak{p}}\left(d_{P}\right)$ is odd, then $v_{\mathfrak{p}}\left(\mathfrak{d}_{K / k}\right)=1$ if $\mathfrak{p} \nmid(2)$ and $v_{\mathfrak{p}}\left(\mathfrak{d}_{K / k}\right)=$ $1+2 e_{0}$ if $\mathfrak{p} \mid(2)$.

Step 3. Else $\left(v_{\mathfrak{p}}\left(d_{P}\right)\right.$ even), if $\mathfrak{p} \nmid(2)$, then $v_{\mathfrak{p}}\left(\mathfrak{d}_{K / k}\right)=0$.

Step 4. Else $\left(v_{\mathfrak{p}}\left(d_{P}\right)\right.$ even and $\left.\mathfrak{p} \mid(2)\right)$ write $d_{P}=\pi^{2 l} x$. If $x \neq \square \bmod \mathfrak{p}^{2}$, then $v_{\mathfrak{p}}\left(\mathfrak{d}_{K / k}\right)=2 e_{0}$. Else, if $x=\square \bmod \mathfrak{p}^{2}$ and $e_{0}=1$, then $v_{\mathfrak{p}}\left(\mathfrak{d}_{K / k}\right)=0$.

Step 5. Else $\left(x=\square \bmod \mathfrak{p}^{2}\right.$ and $\left.e_{0} \neq 1\right)$, if $x \neq \square \bmod \mathfrak{p}^{4}$, then $v_{\mathfrak{p}}\left(\mathfrak{d}_{K / k}\right)=2$ if $e_{0}=2$, and $v_{\mathfrak{p}}\left(\mathfrak{d}_{K / k}\right)=4$ if $e_{0}=3$. Else, if $x=\square \bmod \mathfrak{p}^{4}$ and $e_{0}=2$, then $v_{\mathfrak{p}}\left(\mathfrak{d}_{K / k}\right)=0$.

Step 6. Else $\left(x=\square \bmod \mathfrak{p}^{4}\right.$ and $\left.e_{0}=3\right)$, if $x \neq \square \bmod \mathfrak{p}^{6}$, then $v_{\mathfrak{p}}\left(\mathfrak{d}_{K / k}\right)=2$, else $v_{\mathfrak{p}}\left(\mathfrak{d}_{K / k}\right)=0$.

Note that this algorithm needs to know if $x$ is or is not congruent to a square $\bmod \mathfrak{p}^{2}$, or $\mathfrak{p}^{4}$, or $\mathfrak{p}^{6}$. This is done by use of $\mathfrak{p}$-adic computation depending on the degree of $\mathfrak{p}$.

\section{QUADRATIC SUBFIELDS AND ISOMORPHISMS}

Now, we have tables of sextic fields containing a cubic field, with their relative discriminants. Those fields with a quadratic subfield are in the tables mentioned earlier (see [3]). So, we have to detect whether $K$ contains a quadratic subfield $\tilde{k}$; if the answer is positive, we eliminate those sextic fields from the tables. Next, for sextic fields having the same discriminant, we have to test for $\mathbb{Q}$ isomorphism.

Quadratic subfields. If there exists a quadratic subfield $\tilde{k}=\mathbb{Q}(\sqrt{m})$, then $K$ is the compositum $k \cdot \tilde{k}$ and the polynomials $P(x)$ and $x^{2}-m$ define $K / k$; therefore, the algebraic integer $N_{k / \mathbb{Q}}\left(d_{P}\right) d_{P}$ is a square in $\mathbb{Z}_{k}$ and the converse is true. This assertion gives an algorithm to test whether $\tilde{k}$ exists or not.

Otherwise, if we know approximations to the roots of $P(x)$, there exists a $\tilde{k}$ if and only if there is a partition $\left\{\theta_{1}, \theta_{i}^{\prime}, \theta_{j}^{\prime \prime}\right\}$ and $\left\{\theta_{2}, \theta_{3-i}^{\prime}, \theta_{3-j}^{\prime \prime}\right\}$ (for $i$ and $j$ among 1 and 2$)$ of the six conjugates of $\theta$ such that $\tilde{k}=\mathbb{Q}\left(s_{1}, s_{2}, s_{3}\right)$ or $\tilde{k}=\mathbb{Q}\left(\sqrt{d_{k}}\right),\left(s_{1}, s_{2}, s_{3}\right)$ being the elementary symmetric functions of $\left(\theta_{1}, \theta_{i}^{\prime}, \theta_{j}^{\prime \prime}\right)$.

Consequently, this provides another method for testing if there is a quadratic subfield: for all possible permutations of $\{1,2\}$, we calculate approximations of the $s_{i}$ 's and we test if the $s_{i}$ 's are quadratic integers.

Isomorphisms. We give here two methods for testing the existence of a $\mathbb{Q}$ isomorphism between sextic fields with the same discriminant.

First we refer to $[3, \S 5]$ for a general method; in our case, let $K=k(\theta)$ and $L=k(\varphi)$ be two sextic fields containing $k$ and defined by polynomials $P$ and $Q$ in $\mathbb{Z}_{k}[x]$. Note that since neither $K$ nor $L$ contains a quadratic field, the 
cubic subfield $k$ is unique. Thus, $K$ and $L$ are isomorphic if and only if there exists a permutation $\sigma$ of $\{1,2\}$ such that for $h=0,1$ the sums

$$
\alpha_{h}=\sum_{1 \leq i \leq 2} \theta_{i}^{h} \varphi_{\sigma(i)}, \quad \alpha_{h}^{\prime}=\sum_{1 \leq i \leq 2} \theta_{i}^{\prime h} \varphi_{\sigma(i)}^{\prime}, \quad \alpha_{h}^{\prime \prime}=\sum_{1 \leq i \leq 2} \theta_{i}^{\prime \prime h} \varphi_{\sigma(i)}^{\prime \prime}
$$

belong respectively to $k$ and its conjugates. A numerical computation with sufficient accuracy allows us to decide this effectively.

The second method is based on the following observation: if $K$ and $L$ are $\mathbb{Q}$ isomorphic, they are $k$-isomorphic unless $K$ is cyclic; in this last situation, we need to take into account the possible conjugacies. Therefore, $K=k(\sqrt{\lambda})$ and $L=k(\sqrt{\mu})$ are isomorphic if and only if $\lambda / \mu$ is in $k^{2}$, i.e., $N_{k / \mathbb{Q}}(\lambda) / N_{k / \mathbb{Q}}(\mu)$ is a square in $\mathbb{Q}$.

\section{GALOIS GROUPS}

One can find in [4] the sixteen possible transitive permutation groups of degree six which may be associated with each sextic field. Among these sixteen groups, only five correspond to a sextic field with a cubic subfield and no quadratic subfield (two of them are even).

We give in the table below all such groups for $K=k(\sqrt{\lambda})$, according to the permutation group defined by the cubic subfield $k$ ( $C_{n}$ is the cyclic group of order $n, S_{n}$ is the symmetric group on $n$ letters, and $A_{n}$ is the subgroup of even permutations in $\left.S_{n}\right)$, and the degree of $k_{1}=\mathbb{Q}\left(\sqrt{N_{k / \mathbb{Q}}(\lambda)}\right)$ and $k_{2}=$ $\mathbb{Q}\left(\sqrt{d_{k}}\right)$ over $\mathbb{Q}$.

\begin{tabular}{|c|c|c|l|}
\hline type of $K$ & type of $k$ & $k_{1}, k_{2}$ & possible $r_{1}$ 's \\
\hline$A_{4}$ & $C_{3}$ & $k_{1}=k_{2}=\mathbb{Q}$ & 6,2 \\
$A_{4} \times C_{2}$ & $C_{3}$ & {$\left[k_{1}: \mathbb{Q}\right]=2, k_{2}=\mathbb{Q}$} & $6,4,2,0$ \\
\hline$S_{4}^{+}$ & $S_{3}$ & $k_{1}=\mathbb{Q},\left[k_{2}: \mathbb{Q}\right]=2$ & $6,2(*)$ \\
$S_{4}^{-}$ & $S_{3}$ & $k_{1}=k_{2},\left[k_{1}: \mathbb{Q}\right]=2$ & $6,2,0$ \\
$S_{4} \times C_{2}$ & $S_{3}$ & {$\left[k_{1}: \mathbb{Q}\right]=\left[k_{2}: \mathbb{Q}\right]=2$} & $6,4,2,0(* *)$ \\
\hline
\end{tabular}

(*) two possible Frobenius substitutions when $r_{1}=2$

(**) idem when $r_{1}=2$ or 0 .

$S_{4}^{+}$(resp. $S_{4}^{-}$) denotes the even (resp. odd) permutation of $S_{4}$ on six letters.

By examining the above table we can easily deduce an algorithm to compute the type of $K / \mathbb{Q}$ :

If $k$ is of type $C_{3}$, then the type of $K$ is $A_{4}$ if $N_{k / \mathbb{Q}}(\lambda)$ is a square, and $A_{4} \times C_{2}$ if not.

If $k$ is of type $S_{3}$, then the type of $K$ is $S_{4}^{+}$if $N_{k / \mathbb{Q}}(\lambda)$ is a square, $S_{4}^{-}$if $N_{k / \mathbb{Q}}(\lambda) / d_{k}$ is a square, and $S_{4} \times C_{2}$ otherwise. 
Actually, the effective computation is simply done using the following computational trick: We write $d_{k}=d \cdot f^{2}$ in such a way that $d=1$ means that the type of $k$ is $C_{3}$ (if $d_{k}=m g^{2}$ with $m$ squarefree, then $d=m$ and $f=g$ if $m \equiv 1 \bmod 4$, and $d=4 m, f=g / 2$ in the other cases). To conclude, it suffices to know $N_{k / \mathbb{Q}}\left(d_{P}\right)$; if $d=1$, then the type of $K$ is $A_{4}$ when $N_{k / \mathbb{Q}}\left(d_{P}\right)$ is a square, otherwise $A_{4} \times C_{2}$; if $d \neq 1$, then the type is $S_{4}^{+}$if $N_{k / \mathbb{Q}}\left(d_{P}\right)$ is a square, else $S_{4}^{-}$if $N_{k / \mathbb{Q}}\left(d_{P}\right) / d$ is a square, and $S_{4} \times C_{2}$ otherwise.

\section{A LOOK AT THE TABLES}

The algorithms were implemented on a "sparc-station 1."

The bounds for the discriminants depend only on the signature and on the running time of the algorithms; intensive use of the multiprecision package "PARI" allowed us to avoid being concerned about the size of the integers (these may be about 17 decimal digits in the case of polynomial discriminants).

We chose $M=70,000,000$ (resp. 20,000,000, 16,000,000, 3,000,000, $50,000,000$ and $3,000,000$ ) for $r 1=6$ (resp. 4, 2 and totally real cubic subfield, 2 and complex cubic subfield, 0 and totally real cubic subfield, and 0 and complex cubic subfield); we found respectively $947,994,850,1448,724$, and 1548 sextic fields for $27771,20434,16908,15100,18296$, and 17080 irreducible polynomials with suitable signature. On the workstation mentioned above, the cpu-running times were respectively $101,27,23,11,68$, and 12 minutes.

Distribution of the sextic fields according to Galois type. We give below the distribution of these fields according to signature and Galois type.

\begin{tabular}{|l|r||r|r||r|r|r|}
\hline sign. & \multicolumn{1}{|c||}{$\left|d_{K}\right|$ max. } & \multicolumn{1}{|c||}{$A_{4}$} & \multicolumn{1}{|c|}{$A_{4} \times C_{2}$} & \multicolumn{1}{|c|}{$S_{4}^{+}$} & \multicolumn{1}{c|}{$S_{4}^{-}$} & \multicolumn{1}{|c|}{$S_{4} \times C_{2}$} \\
\hline \hline 6 & 69948333 & 6 & 507 & 45 & 3 & 386 \\
4 & 19983523 & $\times$ & 470 & $\times$ & $\times$ & 524 \\
$2(r)$. & 15981056 & 16 & 359 & 131 & 8 & 336 \\
$2(i)$. & 2999824 & $\times$ & $\times$ & 113 & $\times$ & 1335 \\
$0(r)$. & 49843600 & $\times$ & 370 & $\times$ & $\times$ & 354 \\
$0(i)$. & 2999959 & $\times$ & $\times$ & $\times$ & 29 & 1519 \\
\hline
\end{tabular}

(" $\times$ " means "impossible"; $r$. ( resp. $i$.) points out that the cubic subfield is totally real (resp. complex).)

Note that the norm of the $\mathbb{Z}_{k}$-index of $\mathbb{Z}_{k}[\theta]$ in $\mathbb{Z}_{K}$ which we found is equal to 1 most of the time; the number of exceptions is 30 (resp. 21, 45, 30, 16, and 31 ) for $r_{1}=6$ (resp. 4, 2 and totally real cubic subfield, 2 and complex cubic subfield, 0 and totally real cubic subfield, 0 and complex cubic subfield). 
Minimal discriminants. In the next table, we give the minimal discriminant of sextic fields containing a cubic subfield but no quadratic subfield for each signature and each possible type.

\begin{tabular}{|c||c|c|c|c|}
\hline sign. & $(6,0)$ & $(4,1)$ & $(2,2)$ & $(0,3)$ \\
\hline \hline type & & & 153664 & $\times$ \\
$A_{4}$ & 25969216 & $\times$ & 31213 & -400967 \\
$A_{4} \times C_{2}$ & 434581 & -103243 & & \\
\hline$S_{4}^{+}$ & 3356224 & $\times$ & $\left\{\begin{array}{rr}r . & 52441 \\
i . & 33856\end{array}\right.$ & $\times$ \\
$S_{4}^{-}$ & 33076161 & $\times$ & 810448 & -85184 \\
$S_{4} \times C_{2}$ & 1387029 & -309123 & $\left\{\begin{array}{rr}r . & 109520 \\
i . & 28037\end{array}\right.$ & $\left\{\begin{array}{rr}r . & -503792 \\
i . & -10051\end{array}\right.$ \\
\hline
\end{tabular}

(“ $x$ " means "impossible".)

Coincidences of discriminants. Finally, we show the coincidences of discriminants that are in each of the six tables of sextic fields (i.e., the number of systems of two (resp. three, four, five, and six) nonisomorphic sextic fields with the same discriminants).

\begin{tabular}{|c||r|r|r|r|r|r|}
\hline \multicolumn{1}{|c||}{$r_{1}$} & \multicolumn{1}{c|}{6} & \multicolumn{1}{c|}{4} & \multicolumn{1}{c|}{$2(r)}$. & $2(i)$. & $0(r)$. & \multicolumn{1}{c|}{$0(i)}$. \\
\hline \hline 2 fields & 9 & 144 & 116 & 147 & 8 & 155 \\
3 fields & 3 & 44 & 27 & 48 & 2 & 57 \\
4 fields & 0 & 3 & 3 & 2 & 0 & 0 \\
5 fields & 0 & 2 & 0 & 0 & 0 & 2 \\
6 fields & 0 & 0 & 0 & 2 & 0 & 0 \\
\hline
\end{tabular}

Remarks on class numbers. Let $k_{3}$ be a cubic field; the discriminant of an $A_{4}$ or $S_{4}$ extension (say $k_{4}$ ) attached to $k_{3}$ is $d_{k_{4}}=d_{k_{3}} N_{k_{3} / \mathbb{Q}}\left(\mathfrak{d}_{k_{6} / k_{3}}\right)$, where $k_{6}$ is an $A_{4}$ or $S_{4}^{+}$sextic field ( $A_{4}$ when $k_{3} / \mathbb{Q}$ is cyclic, $S_{4}^{+}$otherwise). The signature of $k_{4}$ is given by the following rules: either $k_{6} / k_{3}$ is unramified at infinity, and then $k_{4}$ is totally real if $k_{3}$ is totally real, and of mixed signature if $k_{3}$ is complex, or $k_{3}$ is totally real, two infinite primes of $k_{3}$ ramify in $k_{6} / k_{3}$, and $k_{4}$ is totally imaginary. The equality $d_{k_{4}}=d_{k_{3}}$ holds if and only if $k_{6} / k_{3}$ is unramified for finite primes. Thus, we recover from the three tables for the signature $(6,0)$ and $(2,2)$, the well-known lists of coincidences between quartic 
and cubic discriminants: $1957,2777, \ldots,-283,-331, \ldots, 229,257, \ldots$; our results are in accordance with Godwin's.

We can describe the corresponding extensions $k_{6} / k_{3}$ by class field theory. When the involved $S_{4}$ field is not totally imaginary, the extensions $k_{6} / k_{3}$ are in one-to-one correspondence with the subgroups of index 2 in $\mathscr{C} l_{k_{3}}$ when $k_{3}$ is not cyclic, and of index 4 and quotient $C_{2} \times C_{2}$ when $k_{3}$ is cyclic. Examples of $S_{4}^{+}$extensions appear in the tables, but the smallest $A_{4}$ example has a discriminant $\left(163^{4}=705,911,761\right)$ which lies beyond the limit of our tables. When the $S_{4}$ field is totally imaginary, we must consider subgroups of $\mathscr{C} l_{k_{3}}^{+}$ which are not pull-backs of a subgroup of $\mathscr{C} l_{k_{3}}$. This is possible if and only if the 2-rank of $\mathscr{C} l_{k_{3}}^{+}$is larger than the 2-rank of $\mathscr{C} l_{k_{3}}$. This never happens when $k_{3}$ is cyclic, and we therefore cannot find $A_{4}$ examples, but $S_{4}^{+}$examples can be found in our tables.

Excerpts of the tables. We conclude with some short excerpts from the tables. Complete tables can be obtained from the author. They are available on floppy disk (source $\mathrm{T}_{\mathrm{E}} \mathrm{X}$ ) or on paper (353 pages) (contact the author by e-mail).

For each of the six tables, we give the first ten sextic fields. The seven columns provide the following data: $d_{K}, d_{k}, N_{k / \mathbb{Q}}\left(\mathfrak{d}_{K / k}\right)$, the Galois group of a Galois closure of $K / \mathbb{Q}, f=N_{k / \mathbb{Q}}(\mathfrak{f})$ such that $d_{P}=\mathfrak{f}^{2} \mathfrak{d}_{K / k}$, a polynomial $P$ which defines $K / k$, and finally $d_{P}$.

The coefficients of the polynomial $P$ are in $\mathbb{Z}_{k}$; so for all the cubic fields which appear in the second column of the tables, we give below a polynomial which defines $k / \mathbb{Q}$ and an integral basis for $\mathbb{Z}_{k}$ of the form $(1, \alpha, \beta), \beta$ being a quadratic polynomial in $\alpha$.

Signature $(6,0)$

$\begin{array}{lrrllll}434581 & 49 & 181 & A_{4} \times C_{2} & 1 & x^{2}-\alpha x+(1+\alpha-3 \beta) & -4-4 \alpha+13 \beta \\ 703493 & 49 & 293 & A_{4} \times C_{2} & 1 & x^{2}-(1+\alpha) x+(-1+\alpha) & 5-2 \alpha+\beta \\ 905177 & 49 & 377 & A_{4} \times C_{2} & 1 & x^{2}-\beta x+(-1-2 \alpha-\beta) & 3+7 \alpha+7 \beta \\ 1279733 & 49 & 533 & A_{4} \times C_{2} & 1 & x^{2}-\beta x+(-4-11 \alpha-5 \beta) & 15+43 \alpha+23 \beta \\ 1292517 & 81 & 197 & A_{4} \times C_{2} & 1 & x^{2}-\alpha x+(3+7 \alpha-5 \beta) & -12-28 \alpha+21 \beta \\ 1387029 & 257 & 21 & S_{4} \times C_{2} & 1 & x^{2}-(1+\alpha+\beta) x+(1+2 \alpha+\beta) & 3+7 \alpha+4 \beta \\ 1397493 & 81 & 213 & A_{4} \times C_{2} & 1 & x^{2}-(1+\alpha+\beta) x+(2+10 \alpha-3 \beta) & -5-31 \alpha+18 \beta \\ 1528713 & 81 & 233 & A_{4} \times C_{2} & 1 & x^{2}-(1+\alpha+\beta) x+(4+10 \alpha-4 \beta) & -13-31 \alpha+22 \beta \\ 1683101 & 49 & 701 & A_{4} \times C_{2} & 1 & x^{2}-(\alpha+\beta) x+(-13+4 \alpha+6 \beta) & 53-13 \alpha-22 \beta \\ 1997632 & 49 & 832 & A_{4} \times C_{2} & 1 & x^{2}+(3+6 \alpha-7 \beta) & -12-24 \alpha+28 \beta\end{array}$

Signature $(4,1)$

$\begin{array}{lrllll}-103243 & 49 & -43 & A_{4} \times C_{2} & 1 & x^{2}-(1+\alpha) x+(-19+5 \alpha+9 \beta) \\ -124659 & 81 & -19 & A_{4} \times C_{2} & 1 & x^{2}-\alpha x+(-1-4 \alpha-2 \beta) \\ -153664 & 49 & -64 & A_{4} \times C_{2} & 1 & x^{2}+(1-3 \alpha-2 \beta) \\ -170471 & 49 & -71 & A_{4} \times C_{2} & 1 & x^{2}-(1+\alpha+\beta) x+(-3+3 \alpha+3 \beta) \\ -199283 & 49 & -83 & A_{4} \times C_{2} & 1 & x^{2}-(1+\alpha+\beta) x+3 \alpha \\ -218491 & 49 & -91 & A_{4} \times C_{2} & 1 & x^{2}-(1+\alpha+\beta) x+(-2-2 \alpha+5 \beta) \\ -304927 & 49 & -127 & A_{4} \times C_{2} & 1 & x^{2}-\beta x+(2+4 \alpha-4 \beta) \\ -309123 & 321 & -3 & S_{4} \times C_{2} & 1 & x^{2}-(1+\alpha) x+(-3+\alpha+\beta) \\ -333739 & 49 & -139 & A_{4} \times C_{2} & 1 & x^{2}-(\alpha+\beta) x+(19-4 \alpha-8 \beta) \\ -334611 & 81 & -51 & A_{4} \times C_{2} & 1 & x^{2}-\alpha x+(-1-3 \alpha-\beta)\end{array}$

$$
\begin{aligned}
& 77-18 \alpha-35 \beta \\
& 4+16 \alpha+9 \beta \\
& -4+12 \alpha+8 \beta \\
& 14-7 \alpha-8 \beta \\
& 2-7 \alpha+4 \beta \\
& 10+13 \alpha-16 \beta \\
& -9-17 \alpha+19 \beta \\
& 13-2 \alpha-3 \beta \\
& -75+19 \alpha+34 \beta \\
& 4+12 \alpha+5 \beta
\end{aligned}
$$


Signature $(2,2)$ on a totally real cubic field

$\begin{array}{lrrclll}31213 & 49 & 13 & A_{4} \times C_{2} & 1 & x^{2}-x+(-4+\alpha+2 \beta) & 17-4 \alpha-8 \beta \\ 52441 & 229 & 1 & S_{4}^{+} & 1 & x^{2}-x-\alpha & 1+4 \alpha \\ 66049 & 257 & 1 & S_{4}^{+} & 1 & x^{2}-(1+\alpha+\beta) x+(2+4 \alpha+2 \beta) & -1-\alpha \\ 69629 & 49 & 29 & A_{4} \times C_{2} & 1 & x^{2}-(1+\alpha) x+(-1-3 \alpha-\beta) & 5+14 \alpha+5 \beta \\ 87616 & 148 & 4 & S_{4}^{+} & 1 & x^{2}-(1+\alpha) x+(2 \alpha+\beta) & 1-6 \alpha-3 \beta \\ 98441 & 49 & 41 & A_{4} \times C_{2} & 1 & x^{2}-(1+\beta) x+(1+\alpha) & -4-5 \alpha+5 \beta \\ 109520 & 148 & 5 & S_{4} \times C_{2} & 1 & x^{2}-(1+\alpha+\beta) x+(4+\alpha) & -14+2 \alpha+5 \beta \\ 111537 & 81 & 17 & A_{4} \times C_{2} & 1 & x^{2}-(1+\beta) x+(-5-4 \alpha+5 \beta) & 21+17 \alpha-15 \beta \\ 142805 & 169 & 5 & A_{4} \times C_{2} & 1 & x^{2}-x+(3+2 \alpha) & -11-8 \alpha \\ 153664 & 49 & 64 & A_{4} & 1 & x^{2}+(-2 \alpha-\beta) & 8 \alpha+4 \beta\end{array}$

Signature $(2,2)$ on a complex cubic field

$\begin{array}{lrrclll}28037 & -23 & 53 & S_{4} \times C_{2} & 1 & x^{2}-\alpha x+(-1-2 \alpha-\beta) & 4+8 \alpha+5 \beta \\ 32269 & -23 & 61 & S_{4} \times C_{2} & 1 & x^{2}-(1+\beta) x+(1-\beta) & -4+\alpha+7 \beta \\ 33856 & -23 & 64 & S_{4}^{+} & 1 & x^{2}+(-2-2 \alpha-\beta) & 8+8 \alpha+4 \beta \\ 35557 & -31 & 37 & S_{4} \times C_{2} & 1 & x^{2}-(1+\alpha) x+1 & -3+2 \alpha+\beta \\ 40733 & -23 & 77 & S_{4} \times C_{2} & 1 & x^{2}-(\alpha+\beta) x+(-5-7 \alpha-4 \beta) & 21+29 \alpha+16 \beta \\ 44965 & -23 & 85 & S_{4} \times C_{2} & 1 & x^{2}-(1+\alpha+\beta) x+(-1-\alpha) & 6+7 \alpha+2 \beta \\ 47081 & -23 & 89 & S_{4} \times C_{2} & 1 & x^{2}-(1+\alpha) x+(-2+2 \alpha+2 \beta) & 9-6 \alpha-7 \beta \\ 50933 & -31 & 53 & S_{4} \times C_{2} & 1 & x^{2}-(1+\alpha+\beta) x+(1+\beta) & 3 \alpha+2 \beta \\ 53429 & -23 & 101 & S_{4} \times C_{2} & 1 & x^{2}-\alpha x+(1-\alpha-\beta) & -4+4 \alpha+5 \beta \\ 56144 & -44 & 29 & S_{4} \times C_{2} & 1 & x^{2}-\beta x+\alpha & 1-2 \alpha+2 \beta\end{array}$

Signature $(0,3)$ on a totally real cubic field

$\begin{array}{lrlllll}-400967 & 49 & -167 & A_{4} \times C_{2} & 1 & x^{2}-\beta x+3 & -13-\alpha+3 \beta \\ -465831 & 81 & -71 & A_{4} \times C_{2} & 1 & x^{2}-x+(2+\alpha) & -7-4 \alpha \\ -503792 & 148 & -23 & S_{4} \times C_{2} & 1 & x^{2}-x+(4-\alpha-\beta) & -15+4 \alpha+4 \beta \\ -573839 & 49 & -239 & A_{4} \times C_{2} & 1 & x^{2}-(1+\alpha+\beta) x+4 & -14+5 \alpha+4 \beta \\ -602651 & 49 & -251 & A_{4} \times C_{2} & 1 & x^{2}-\alpha x+(2-5 \alpha+3 \beta) & -8+20 \alpha-11 \beta \\ -679024 & 148 & -31 & S_{4} \times C_{2} & 1 & x^{2}-x+(-\alpha+\beta) & 1+4 \alpha-4 \beta \\ -839056 & 229 & -16 & S_{4} \times C_{2} & 1 & x^{2}-(1+\alpha+\beta) x+(1+4 \alpha+3 \beta) & -1-5 \alpha-5 \beta \\ -909979 & 49 & -379 & A_{4} \times C_{2} & 1 & x^{2}-(1+\alpha+\beta) x+(1+4 \alpha+5 \beta) & -2-11 \alpha-16 \beta \\ -1142512 & 404 & -7 & S_{4} \times C_{2} & 1 & x^{2}-\alpha x+(4+2 \alpha) & -16-8 \alpha+\beta \\ -1178891 & 49 & -491 & A_{4} \times C_{2} & 1 & x^{2}-\alpha x+(1-\alpha+\beta) & -4+4 \alpha-3 \beta\end{array}$

Signature $(0,3)$ on a complex cubic field

$\begin{array}{lllllll}-10051 & -23 & -19 & S_{4} \times C_{2} & 1 & x^{2}-(1+\alpha) x+1 & -3+2 \alpha+\beta \\ -10571 & -31 & -11 & S_{4} \times C_{2} & 1 & x^{2}-(1+\beta) x+(1+\beta) & -2+\alpha-\beta \\ -18515 & -23 & -35 & S_{4} \times C_{2} & 1 & x^{2}-(\alpha+\beta) x+(-2+4 \alpha-\beta) & 9-15 \alpha+4 \beta \\ -22747 & -23 & -43 & S_{4} \times C_{2} & 1 & x^{2}-(1+\alpha) x+(3+4 \alpha+2 \beta) & -11-14 \alpha-7 \beta \\ -27556 & -83 & -4 & S_{4} \times C_{2} & 1 & x^{2}-\beta x+1 & -2+\alpha \\ -27848 & -59 & -8 & S_{4} \times C_{2} & 1 & x^{2}-(1+\alpha) x+(2+\alpha+\beta) & -7-2 \alpha-3 \beta \\ -29095 & -23 & -55 & S_{4} \times C_{2} & 1 & x^{2}-(\alpha+\beta) x+(1+2 \alpha+\beta) & -3-7 \alpha-4 \beta \\ -31211 & -23 & -59 & S_{4} \times C_{2} & 1 & x^{2}-x+\alpha & 1-4 \alpha \\ -33856 & -23 & -64 & S_{4} \times C_{2} & 1 & x^{2}+\alpha & -4 \alpha \\ -37479 & -31 & -39 & S_{4} \times C_{2} & 1 & x^{2}-(1+\alpha+\beta) x+(-1-3 \alpha+5 \beta) & 8+15 \alpha-14 \beta\end{array}$




\section{Totally real cubic fields}

$d_{k} \quad$ ind. polyn. of $\alpha$

$\begin{array}{rll}49 & 1 & x^{3}+x^{2}-2 x-1 \\ 81 & 1 & x^{3}-3 x-1 \\ 148 & 1 & x^{3}+x^{2}-3 x-1 \\ 169 & 1 & x^{3}-x^{2}-4 x-1 \\ 229 & 1 & x^{3}-4 x-1 \\ 257 & 1 & x^{3}-5 x-3 \\ 321 & 1 & x^{3}+x^{2}-4 x-1 \\ 404 & 1 & x^{3}-x^{2}-5 x-1\end{array}$

integ. basis

$$
\begin{aligned}
& \left(1, \alpha, \alpha^{2}\right) \\
& \left(1, \alpha, \alpha^{2}\right) \\
& \left(1, \alpha, \alpha^{2}\right) \\
& \left(1, \alpha, \alpha^{2}\right) \\
& \left(1, \alpha, \alpha^{2}\right) \\
& \left(1, \alpha, \alpha^{2}\right) \\
& \left(1, \alpha, \alpha^{2}\right) \\
& \left(1, \alpha, \alpha^{2}\right)
\end{aligned}
$$

Complex cubic fields

$\begin{array}{llll}-23 & 1 & x^{3}+x^{2}-1 & \left(1, \alpha, \alpha^{2}\right) \\ -31 & 1 & x^{3}-x^{2}-1 & \left(1, \alpha, \alpha^{2}\right) \\ -44 & 1 & x^{3}-x^{2}-x-1 & \left(1, \alpha, \alpha^{2}\right) \\ -59 & 1 & x^{3}+2 x^{2}-1 & \left(1, \alpha, \alpha^{2}\right) \\ -83 & 1 & x^{3}-x^{2}+x-2 & \left(1, \alpha, \alpha^{2}\right)\end{array}$

\section{ACKNOWLEDGMENTS}

This work could not have been conducted without the sound advice of $\mathbf{J}$. Martinet. In order to perform the many calculations needed here, we made intensive use of the PARI-package, created under the supervision of $\mathrm{H}$. Cohen; this package is implemented on SUN workstations which are partially supported by the "P.R.C. mathématiques et informatique."

\section{BIBLIOGRAPHY}

1. I. O. Angell, A table of complex cubic fields, Bull. London Math. Soc. 5 (1973), 37-38.

2. __ A table of totally real cubic fields, Math. Comp. 30 (1976), 184-187.

3. A.-M. Bergé, J. Martinet, and M. Olivier, The computation of sextic fields with a quadratic subfield, Math. Comp. 54 (1990), 869-884.

4. G. Butler and J. McKay, The transitive groups of degree up to eleven, Comm. Algebra 11 (1983), 863-911.

5. V. Ennola and R. Turunen, On totally real cubic fields, Math. Comp. 44 (1985), 495-518.

6. U. Fincke and M. Pohst, Improved methods for calculating vectors of short length in a lattice, including a complexity analysis, Math. Comp. 44 (1985), 463-471.

7. H. J. Godwin, The determination of fields of small discriminant with a given subfield, Math. Scand. 6 (1958), 40-46.

8. H. J. Godwin and P. Samet, A table of real cubic fields, J. London Math. Soc. 34 (1959), 108-110.

9. P. Llorente and E. Nart, Effective determination of the decomposition of the rational primes in a cubic field, Proc. Amer. Math. Soc. 87 (1983), 579-585.

10. P. Llorente and J. Quer, On totally real cubic fields with discriminant $D<10^{7}$, Math. Comp. 50 (1988), 581-594.

11. J. Martinet, Méthodes géométriques dans la recherche des petits discriminants, Progr. Math., vol. 59, Birkhäuser, Boston, 1985, pp. 147-179.

12. _ Discriminants and permutation groups, Number Theory (Richard A. Mollin, ed.), Walter de Gruyter, Berlin and New York, 1990, pp. 359-385. 
13. M. Olivier, Corps sextiques primitifs, Ann. Inst. Fourier (Grenoble) 40 (1990), 757-767.

14. M. Pohst, On the computation of number fields of small discriminants including the minimum discriminants of sixth degree fields, J. Number Theory 14 (1982), 99-117.

15. D. Shanks, Review of I. O. Angell, "Table of complex cubic fields", Math. Comp. 29 (1975), Review 33, 661-665.

16. P. Smadja, Calculs effectifs sur les idéaux des corps de nombres algébriques, Univ. d'AixMarseille, U.E.R. de Luminy, 1976.

Centre de Recherche en Mathématiques de Bordeaux, 351 cours de la libération, 33405 TALENCE CEDEX, France

E-mail address: olivier@mizar.greco-prog.fr 